\title{
GPCR allostery and bias in modern pharmacology: Structural, pharmacological and clinical implications
}

\section{Christopher J. Langmead}

Drug Discovery Biology, Monash Institute of Pharmaceutical Sciences, Monash University, Australia

G protein-coupled receptors (GPCRs) constitute the largest class of drug targets, but are associated with high attrition in translating preclinical discoveries into the clinic. In part, this may reflect a failure to appreciate and capture novel paradigms associated with drug action at GPCRs. Indeed, it is now well established that GPCRs possess spatially distinct allosteric sites that can be found at extracellular, transmembrane-spanning or intracellular domains. Moreover, GPCRs are highly dynamic proteins that adopt multiple active states, which can be differentially stabilized by different ligand classes to yield biased agonism resulting in recruitment of a subset of possible signaling pathways while sparing or blocking others. Targeting GPCR allosteric sites has the potential to lead to novel modes of GPCR subtype selectivity, signalpathway-selective (biased) modulation and, importantly, a saturability to the allosteric effect that can be exploited to finetune drug responsiveness in a positive or negative direction. However, many of these theoretical advantages of allosteric drugs have yet to be optimally explored in the context of disease, and translating these concepts into clinical programs represents a significant next step for the field. For instance, allosteric modulators can have different effects on orthosteric ligand affinity relative to signaling efficacy, as well as directly activating the receptor themselves, and it is likely that such differences in mode of action will affect the successful targeting of different disease states with allosteric drugs. Excitingly, structural biology studies are starting to identify the molecular mechanisms that underlie the pharmacological effects of allosteric modulators and are facilitating structure-based allosteric drug discovery at this important receptor family. Finally, chemical biology approaches are generating novel tools for interrogating GPCR structure and manipulating GPCR functionality, including bitopic ligands that concomitantly bridge orthosteric and allosteric sites. Although not often appreciated, it should be noted that many of these properties are likely common to all receptor superfamilies. 